\title{
Parálisis vocal permanente: corrección quirúrgica con tiroplastia tipo I
}

\author{
Permanent vocal paralysis: type I thyroplasty surgical correction
}

\author{
Rubén Yangali ${ }^{1}$, Kattia Moreno ${ }^{2}$ \\ ${ }^{1}$ Cirujano de Cabeza Cuello y Maxilofacial, Servicio de Otorrinolaringología y Cirugía de Cabeza y Cuello. Hospital Central de la Fuerza Aérea del Perú. Lima, Perú. \\ ${ }^{2}$ Research Assistant Otolaryngology and Head and Neck Surgery. University Of Cincinnati, Ohio, USA.
}

\section{Resumen}

La parálisis vocal permanente es causa de disfonía y episodios repetitivos de aspiración. Hacemos una revisión breve de la fisiopatología así como del procedimiento quirúrgico correctivo, conocido como tiroplastia.

Palabras clave: Parálisis de las cuerdas vocales; tiroplastia.
Abstract

Permanent vocal cord paralysis causes dysphonia and repetetive aspiration episodes. We review vocal cord paralysis pathophysiology and the corrective surgical procedure known as thyroplasty.

Key words: Vocal cord paralysis; thyroplasty.

\section{INTRODUCCIÓN}

La parálisis de cuerda vocal se define como la falta de movilidad de una o las dos cuerdas vocales, lo cual es consecuencia de algún tipo de injuria sobre el nervio que inerva la musculatura intralaringea (nervio recurrente laringeo). Esta falta de movilidad de la cuerda vocal hace imposible la aproximación competente de ambas cuerdas vocales, generando como cuadro clínico una disfonía marcada acompañada de episodios aspirativos ${ }^{(1-3)}$.

La parálisis vocal es una entidad mal tolerada por la persona que la padece. No solo la mala calidad de voz sino también los episodios de aspiración repetitivos, con sensación de ahogo que esta produce, generan frustración y limitaciones en las personas y más aún en aquellas que trabajan con la voz (profesores, locutores, recepcionistas), generando incluso problemas laborales o de integración social.

La terapia de rehabilitación vocal, como elemento terapéutico en el manejo de esta entidad, es de vital importancia, básicamente en los casos de etiología idiopática, vírica o inflamatoria, es decir, en los casos en los cuales puede haber una regresión por lo menos parcial de la parálisis vocal ${ }^{(3)}$.

En los casos donde existe evidencia de lesión nerviosa permanente (nervio laríngeo recurrente, tronco vagal o a nivel central), si bien es cierto que la terapia de rehabilitación vocal continúa siendo de gran ayuda, los resultados no son del todo satisfactorios. Es en este contexto en que la tiroplastia cobra importancia.
La tiroplastia, procedimiento quirúrgico que se realiza con anestesia local, busca corregir este defecto, con resultados por demás satisfactorios. Exponemos a continuación una breve revisión del tema de parálisis vocal, así como describimos la técnica quirúrgica de la tiroplastia tipo I.

\section{FISIOPATOLOGÍA}

La emisión de la voz es el resultado complejo y armonioso de una serie de funciones de diferentes órganos (pulmones, bronquios, tráquea, laringe, faringe, boca, senos paranasales, entre otros).

El flujo de aire proveniente de los pulmones a través del árbol bronquial se encuentra con las cuerdas vocales a nivel de la caja laríngea. Este flujo de aire choca con las cuerdas vocales medializadas, provocando su vibración y generando sonido, el cual es modulado por la respiración, los movimientos de la lengua y la boca y encuentra su resonancia en los senos paranasales, emitiéndose un sonido armonioso y particular conocido como voz.

En este complejo sistema, es muy importante la movilidad de las cuerdas vocales, que funcionan a manera de válvula en $\mathrm{V}$ invertida. Durante la emisión de voz y durante la deglución, las cuerdas vocales se cierran (aducción); durante la respiración, se abren (abducción). Figura 1.

Cuando existe parálisis de una cuerda vocal, sea esta derecha o izquierda (o ambas), esta no puede juntarse con la otra en la línea media; por tanto, el flujo de aire proveniente de los pulmones no encuentra resistencia a su salida, las cuerdas vocales no vibran correctamente y se produce una voz deficiente (disfonía). Por otra parte, cuando el individuo deglute líquidos o incluso su propia saliva, al no existir un cierre competente de las cuerdas vocales, estos fluidos pueden discurrir hacia la subglotis o tráquea, generando reflejo tusígeno y sensación de aspiración y ahogo.

La movilidad de las cuerdas vocales se da gracias a la innervación motora del nervio vago sobre la musculatura laríngea. Esta innervación se da a través de sus ramas: el nervio laríngeo inferior (recurrente laríngeo), que es el más importante, ya que inerva la totalidad de músculos laríngeos, excepto el músculo cricotiroideo; y el nervio laringeo superior, que inerva el músculo cricotiroideo, además de dar la sensibilidad endolaringea.

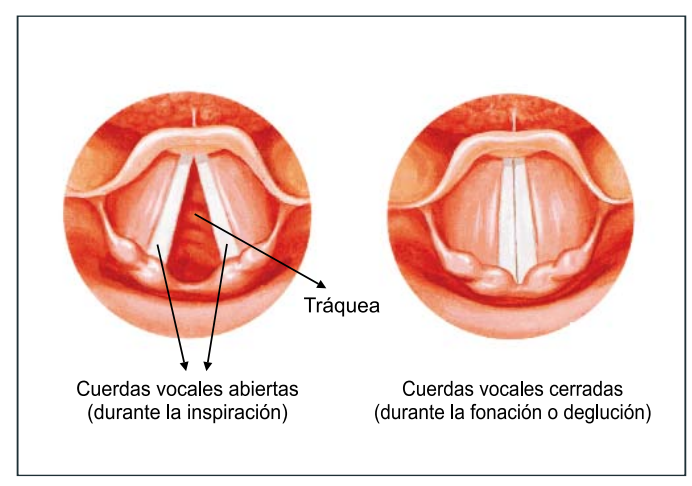

Figura 1. Dinámica de las cuerdas vocales. 
Las parálisis permanentes de cuerda vocal se dan con más frecuencia por lesión del nervio recurrente laríngeo. Este nervio es particularmente susceptible a injuria, ya que tiene un largo trayecto (el izquierdo más que el derecho); es así que, procedimientos quirúrgicos en el tórax, tráquea, esófago, tiroides o tumores en cualquiera de estas áreas pueden afectar de manera permanente a uno o los dos nervios recurrentes laríngeos, generando la sintomatología antes descrita (1-3).

\section{MEDIALIZACIÓN QUIRÚRGICA DE CUERDA VOCAL PARALÍTICA}

Siendo conocida la necesidad de medializar la cuerda vocal paralizada para así mejorar los problemas de disfonía y de aspiración, muchas técnicas quirúrgicas fueron diseñadas para tal fin. Así, la inyección intracordal de colágeno (Ford , 1987) ${ }^{(4)}$ y luego la inyección intracordal de grasa autóloga (Campos,1997) (5) son técnicas que buscan dar mayor volumen a la cuerda vocal paralizada, mejorando el cierre glótico. Los inconvenientes de estas técnicas son la dificultad para lograr una aplicación uniforme y la inevitable reabsorción grasa que sucede en el tiempo, empobreciendo los resultados que inicialmente pudieron ser aceptables.

La tiroplastia tipo I (descrita en sus inicios por Isshiki, 1974) ${ }^{(6,7)}$ es una técnica que se ha ido perfeccionando. En sus inicios, la prótesis de silicona a colocar era tallada manualmente hasta obtener el tamaño adecuado, pero esto demandaba un mayor tiempo operatorio y formas de prótesis no siempre uniformes. Actualmente, y luego de décadas de estudio, se ha estandarizado la forma y las posibles medidas de prótesis, según el sexo del paciente, siendo el material de dicha prótesis silicona de la más alta calidad y biocompatibilidad.

Este procedimiento es de elección en la gran mayoría de casos de parálisis vocal permanente.

\section{Técnica quirúrgica}

La tiroplastia tipo I se realiza con anestesia local y neuroleptoanalgesia, es decir el paciente debe estar despierto y ser capaz de hablar durante el acto quirúrgico. Realizada la asepsia y antisepsia respectiva, se grafica en la piel y con azul de metileno el cartílago tiroideo, cricoides y primeros anillos traqueales. Luego, se infiltra con xilocaína con epinefrina (al 2\%) el área a incidir. Figuras 2 y 3 .

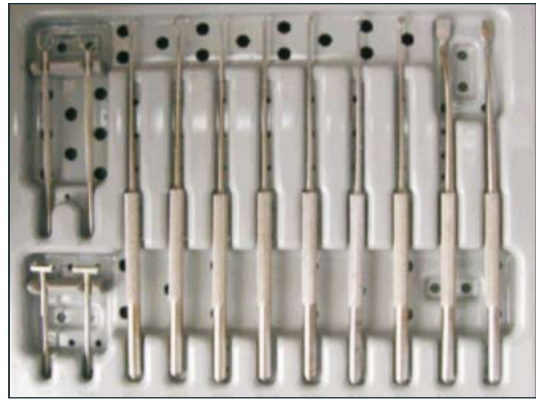

Figura 2. Instrumental básico para tiroplastia.

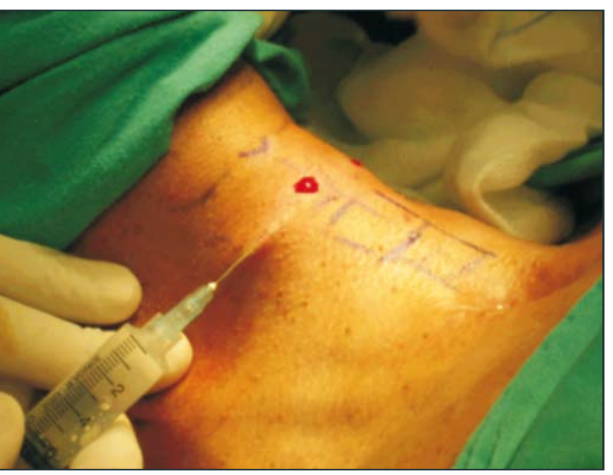

Figura 3. Diseño e infiltración con xilocaína.

Realizada la incisión, se separa el platisma, luego el músculo omohioideo y esternohioideo, se identifica el músculo esternotiroideo en la lámina lateral del cartílago tiroideo; este músculo se secciona, para exponer el tubérculo tiroideo en el reborde inferior del cartílago tiroideo; dicho tubérculo tiroideo es punto de reparo importante para la colocación de un dispositivo conocido como calibrador de ventana (Figura 4), el cual permite diseñar el lugar correcto donde se debe realizar la ventana en el cartílago tiroideo. Graficada el área a incidir con azul de metileno, se procede a abrir una pequeña ventana laríngea (de aproximadamente $7 \times 12 \mathrm{~mm}$, en el varón, y 7×10 mm en la mujer). Esto se realiza con la ayuda de una microsierra. Retirado el fragmento de cartílago, se precede a separar el pericondrio interno de los bordes de la ventana y se corta el pericondrio a nivel de la línea media anterosuperior. Esto eliminará el efecto en carpa que causa una innecesaria medialización superior o inferior a la cuerda vocal. Figura 5.

Luego, se precede a colocar e ir probando del más pequeño al más grande los probadores de ventana (dispositivos de igual tamaño que las prótesis definitivas y que vienen numerados, en general del 6 al 10 para la mujer y del 8 al 12 para el hombre). Figura 7. Para cada prueba, se pide al paciente que hable, simultáneamente se verifica la calidad de voz y con la ayuda de un nasofibroscopio (previa instilación de spray de anestésico en la vía aérea) se verifica la medialización de la cuerda vocal paralizada. Aquella medida de probador de ventana que mejor medialización de cuerda vocal y mejor calidad de voz nos dé, será la medida seleccionada. Luego, se procede a administrar un adicional de analgesia endovenosa y se coloca la prótesis

definitiva, según la medida seleccionada ${ }^{(3,8,9)}$. Figuras 6 y 7 .

Por último, se deja un pequeño drenaje al vacío en el lecho cruento, se sutura el plano músculo aponeurótico con vicryl 4/0 y la piel con nylon 5 ó 6/0.

La cobertura antibiótica, los corticoides y los analgésicos están indicados.

El paciente puede salir de alta al día siguiente, sin drenajes, para controles ambulatorios.

El estudio tomográfico puede estar indicado para verificar la posición correcta de la prótesis. Figura 8.

Al cabo de una semana, se retira los puntos; la cicatriz es imperceptible, ya que se disimula en un pliegue natural (Figura 9: archivos de audio).

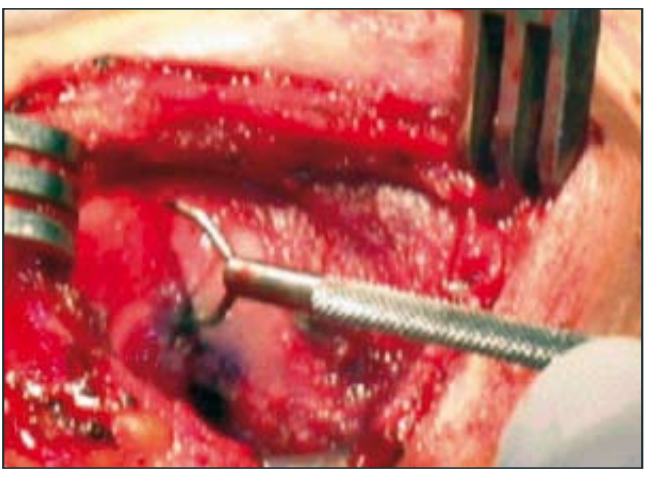

Figura 4. Utilización del calibrador de ventana.

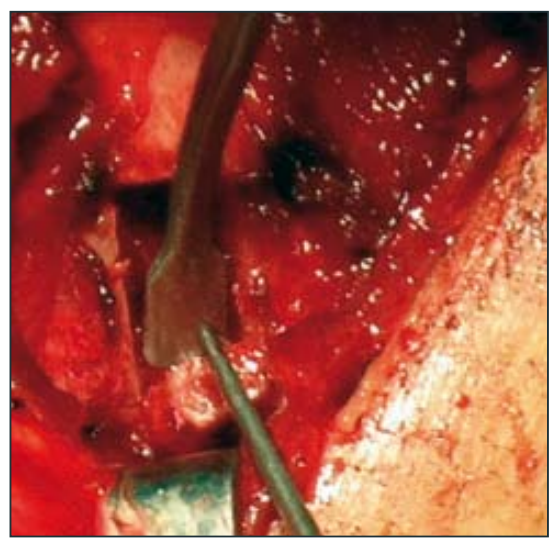

Figura 5. Apertura de la ventana laringea. 


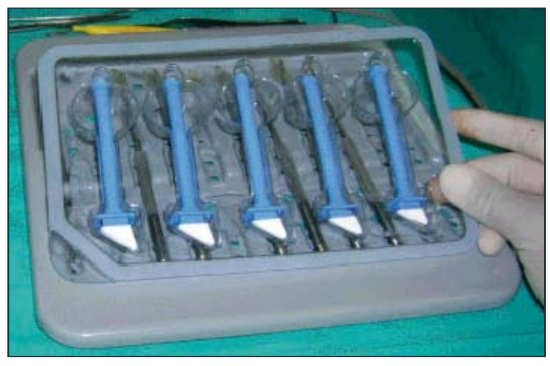

Figura 6. Probadores de ventana

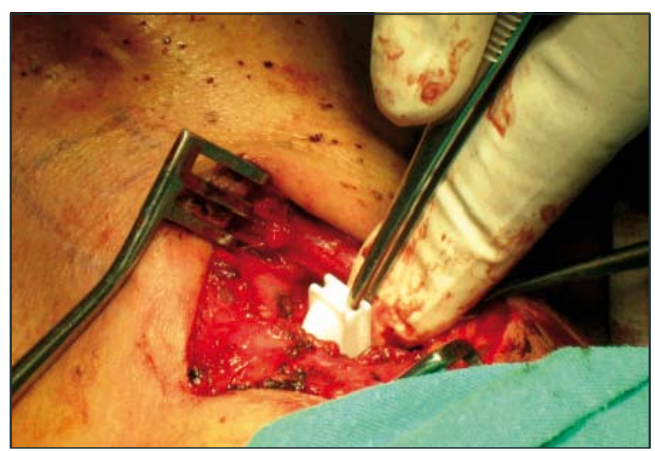

Figura 7. Colocación de prótesis definitiva.

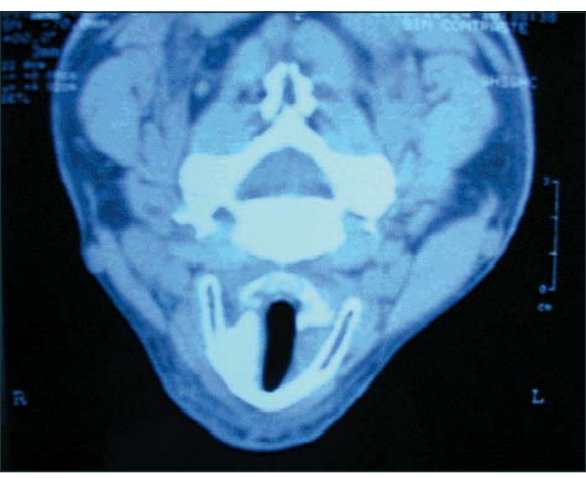

Figura 8. Imagen tomográfica que muestra la prótesis correctamente colocada en la caja laríngea.

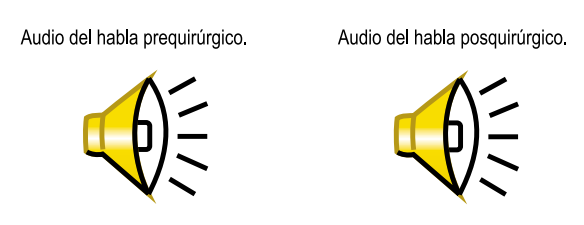

Disponible en: http://www.unmsm.edu.pe/anales/v69/n1

Figura 9. Archivos de audio.

\section{DISCUSIÓN Y COMENTARIOS}

Los resultados de la corrección quirúrgica de la parálisis permanente de cuerda vocal a través de la tiroplastia tipo I son espectaculares; estos son objetivables en el mismo acto quirúrgico.
El procedimiento quirúrgico en sí no demanda mayores riesgos, ya que la caja laríngea es de fácil accesibilidad y los grandes vasos del cuello se mantienen en todo momento alejados del campo quirúrgico. La cirugía no interfiere con el órgano fonatorio en sí, es decir, no se tocan en ningún momento las cuerdas vocales o la mucosa intralaringea. Estas estructuras son respetadas y simplemente son desplazadas por efecto mecánico de la prótesis; de ahí la fácil reversibilidad de la técnica en los casos excepcionales en los que esté indicado el retiro de la prótesis.

$\mathrm{Al}$ ser un procedimiento que se realiza en gran parte de la cirugía con anestesia local, le permite al mismo paciente reconocer la calidad de voz emitida durante la fonación, de tal manera que el paciente participa activamente durante el acto operatorio y en coordinación con el médico, y según la visualización fibroscopica que tenga este, en la determinación de la medida de prótesis más adecuada para el caso.

Es importante señalar que a veces es difícil predecir si una parálisis vocal por lesión nerviosa será permanente o mostrará alguna recuperación. Actualmente se acepta que toda condición de parálisis por injuria nerviosa mayor de un año ya es permanente y por tanto a partir de este momento debe ser considerada una solución quirúrgica a través de la tiroplastia.

Entre los estudios preoperatorios a una tiroplastia están indicados la laringoestroboscopia, la prueba manual de compresión del lado afectado y los tiempos de fonación máximo, los cuales pueden ser repetidos al mes, a los tres meses y a los seis meses, a fin de valorar los resultados quirúrgicos ${ }^{(10-13)}$.

Al ser la tiroplastia tipo I un procedimiento bastante seguro, está indicada en pacientes con diagnósticos oncológicos con parálisis de cuerda vocal, con el fin de mejorar la calidad de vida, ya que esta no altera el pronóstico ni la sobrevida en estos pacientes ${ }^{(14)}$.

Es importante destacar que la terapia de voz debe continuar posterior a la cirugía, ya que esto ayudará a que el paciente adecúe sus nuevas condiciones anatomofisiológicas a la fonación ${ }^{(3)}$

Si bien es cierto que la tiroplastia tipo I es un procedimiento bastante seguro, no está exenta de posibles complicaciones; se ha descrito el eritema, edema y hematoma laríngeo ${ }^{(15-17)}$. Cotter, Avidano y col. ${ }^{(17)}$ clasificaban las posibles complicaciones de una tiroplastia en complicaciones mayores, en las que se incluía a la hemorragia, la obstrucción de la vía aérea y la extrusión de la prótesis, y como complicaciones menores al hematoma de la cuerda vocal, sin obstrucción de la vía aérea, y a la prótesis móvil. Actualmente, el diseño prefabricado de la prótesis que engasta a medida en la caja laringe y ahorra tiempos quirúrgicos, hacen muy eventuales estas posibles complicaciones mayores. De cualquier forma, ante una eventualidad de complicación mayor, el retiro de la prótesis siempre será lo indicado, lo cual llevará a una rápida remisión de los síntomas.

Actualmente, se considera a la tiroplastia tipo I el tratamiento de elección para los casos de parálisis vocal permanente, por parálisis recurrencial ${ }^{(18-21)}$.

\section{REFERENCIAS BIBLIOGRÁFICAS}

1. Abello P. Otorrinolaringología. Barcelona: Edit Doyma; 1992.

2. Paparella. Otorrinolaringología. Buenos Aires: Edit. Panamericana; 1991.

3. Arias C. Parálisis laríngeas. Diagnóstico y tratamiento foniátrico de las parálisis laríngeas cordales unilaterales en abducción. Barcelona: Ed Masson; 1994.

4. Ford C, Bless D. Collagen injection in the scarred vocal fold. J Voice. 1987;1:116-8.

5. Campos G. Tratamiento de la incompetencia glótica mediante lipoinyección bilateral de los pliegues vocales. Acta ORL \& Cir Cabeza y Cuello. 1997;25(2):99-105.

6. Isshiki N. Phonosurgery. Theory and Practice. Tokyo: Springer-Verlag; 1989.

7. Isshiki N, Taira T, Kojima H, Shoji K. Recent modifications in thyroplasty type I. Ann Otol Rhinol Laryngol. 1989;98:777-9.

8. Boston Medical Products. Surgical \& Respiratory Catalog. 2005-2006. Westborough, Massachusetts: Boston Medical Products; c2006. Disponible en: http://www.bosmed.com/ search/search.php?zoom_and=0\&zoom_cat $=$ $1 \& z 0 o m \_p a g e=2 \& z 0 o m \_p e r \_p a g e=10 \& z o o m \_$ query=novatech\&zoom_sort $=0$

9. Boston Medical Products. Educational Materials: Product Catalog and Resource CD. Massachusetts: Boston Medical Products; c2006. Disponible en: http://www.bosmed.com/educational.html

10. Guerrero J, Cobeta I, García-Díaz JD. Cirugía de esqueleto laríngeo. Acta Otorrinolaring Esp. 1998;49(1):45-9.

11. Woo P, Casper J, Colton R, Brewer D. Aerodynamic and stroboscopic findings, before and after microlaryngeal phonosurgery. J Voice. 1994;8:2. 
12. Uloza V, Pribuisiene R, Saferis V. Multidimensional assessment of functional outcomes of medialization thyroplasty. Eur Arch Oto-rhinolaryngol. 2005;262(8):616-21.

13. Núñez F, Suárez C, Llorente J L. Valoración preoperatoria de la tiroplastia: El test de compresión lateral de la laringe. Acta Otorrinolaring Esp. 2001;52:47-51.

14. Lam PK, Ho WK, Ng ML, Wei WI. Medialization thyroplasty for cancer-related unilateral vocal fold paralysis. Otolaryngol Head Neck Surg. 2007;136(3):440-4.

15. Mary M, Gorham, MS, Michael A. Laryngeal recovery following type I thyroplasty. Arch Otolaryngol Head Neck Surg. 1998;124:739-42.

16. Tucker HM, Wanamaker J, Trott M, Hicks D. Complications of laryngeal framework surgery (phonosurgery). Laryngoscope. 1993;103:5258.

17. Cotter CS, Avidano MA, Crary MA, Cassini NJC, Gorham MM. Laryngeal complications after type I thyroplasty. Otolaryngol Head Neck Surg. 1995;113: 671-3.

18. Koufman JA, Isaacson G. Trastornos de la voz. Clínicas Otorrinolaringológicas de Norteamérica. México: Interamericana McGraw; 1991:1023-34. 19. Netterville JL, Stone RE, Luken ES, Civantos FJ, Ossoff RH. Silastic medialization and arytenoid adduction: The Vanderbilt Experience. Ann Otol Rhinol Laryngol. 1993;102:413-24.

20. Sasaki CT, Driscoll BP, Gracco C, Eisen R. The fate of medialized cartilage in thyroplasty type I. Arch Otolaryngol Head Neck Surg. 1994;120:13981399.
21. Montgomery WW, Blaugrund SM, Varvares MA. Thyroplasty: a new approach. Ann Otol Rhinol Laryngol. 1993;102:571-9.

Manuscrito recibido el 10 de enero de 2008 y aceptado para publicación el 15 de febrero de 2008.

\section{Correspondencia:}

Rubén Yangali López

Servicio de Otorrinolaringología y Cirugía de

Cabeza y Cuello. Hospital Central de la

Fuerza Aérea del Perú (FAP)

Av. Aramburú 200, Miraflores

Lima 18, Perú

Correo-e: bengali_r@yahoo.com 